\title{
Thermal Expansion, Response to Pressure, and Reversible Pressure-Induced Amorphization in Anion Excess $\mathrm{ReO}_{3}$-Type Cubic LuZrF7
}

\author{
Anthony J. Lloyd IIt and Angus P. Wilkinson*†§
}

${ }^{+}$School of Chemistry and Biochemistry and ${ }^{\$}$ School of Materials Science and Engineering, Georgia Institute of Technology, Atlanta, Georgia 30332, United States.

*angus.wilkinson@chemistry.gatech.edu, corresponding author

\section{List of Supplementary Figures}

Figure S1. Crystallographic model for cubic $(P m-3 m) L Z_{r F}$ as used in the Rietveld analyses. This model is derived from one of several related models that were explored and reported by Poulain and Tofield ${ }^{1}$ in a structural study of cubic $\mathrm{YbZrF}_{7}$, which made use of single crystal X-ray and neutron powder diffraction data. The Lu and $\mathrm{Zr}$ are statistically disordered and both the cation and anion locations are modeled with split site positions. This heavily disordered model was interpreted by Poulain and Tofield as indicating that the structure consisted of pentagonally bipyramidal coordination polyhedral linked through corners (the 12j F site) and shared edges (pairs of fluorides associated with the $24 \mathrm{l}$ site) ...........2

Figure S2. Rietveld fit to data from the high pressure ( $0.69 \mathrm{GPa}$ ) experiment on cubic $\mathrm{LuZrF}_{7}$. The green tick marks indicate the positions of peaks from the $\mathrm{NaCl}$ pressure standard.

Figure S3. Selected patterns for $\mathrm{LuZrF}_{7}$ on compression. ${ }^{*}$ peaks from the $\mathrm{NaCl}$ pressure calibrant. ........ 2

\section{List of Supplementary Tables}

Table S1. Crystallographic model for the Rietveld fit to the $302 \mathrm{~K}$ ambient pressure data from the Cryostream experiment.

Table S2. Crystallographic model for the Rietveld fit to the $0.35 \mathrm{GPa}$ room temperature data from the high pressure experiment. The structural parameters were fixed at those obtained from the analysis of

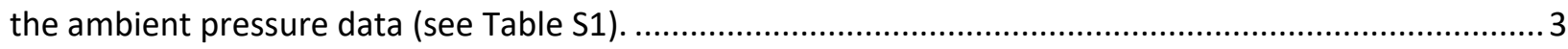

Table S3. Lattice constant and unit cell volume as a function of temperature for cubic $\mathrm{LuZrF}_{7} \ldots \ldots \ldots \ldots \ldots . . . . .4$

Table S4. Lattice constant and unit cell volume as a function of pressure for cubic LuZrF $7 . \ldots \ldots \ldots \ldots \ldots \ldots \ldots . . . . .14$

\section{$\underline{\text { References }}$}

(1) Poulain, M.; Tofield, B. C. The Structure of Cubic YbZrF7. J. Solid State Chem. 1981, 39 (3), 314. 


\section{Supplementary Figures}

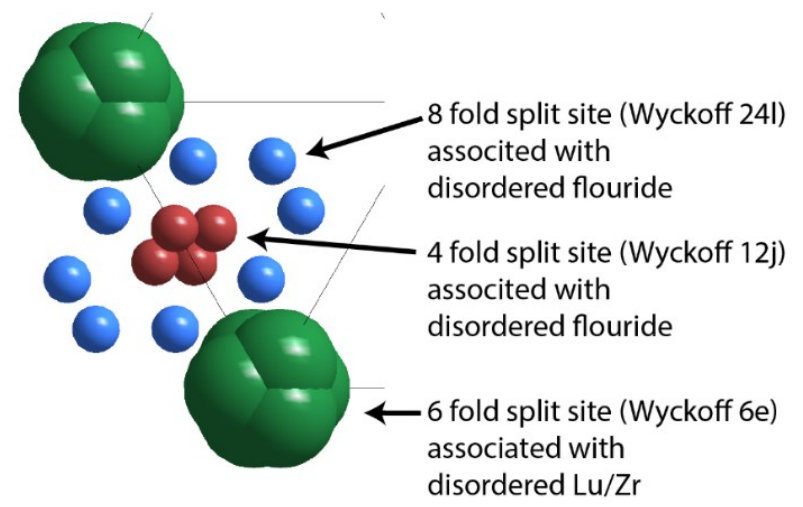

Figure S1. Crystallographic model for cubic $(\mathrm{Pm}-3 \mathrm{~m}) \mathrm{LuZrF}_{7}$ as used in the Rietveld analyses. This model is derived from one of several related models that were explored and reported by Poulain and Tofield ${ }^{1}$ in a structural study of cubic $\mathrm{YbZrF}_{7}$, which made use of single crystal X-ray and neutron powder diffraction data. The Lu and $\mathrm{Zr}$ are statistically disordered and both the cation and anion locations are modeled with split site positions. This heavily disordered model was interpreted by Poulain and Tofield as indicating that the structure consisted of pentagonally bipyramidal coordination polyhedral linked through corners (the $12 \mathrm{j} F$ site) and shared edges (pairs of fluorides associated with the 241 site)

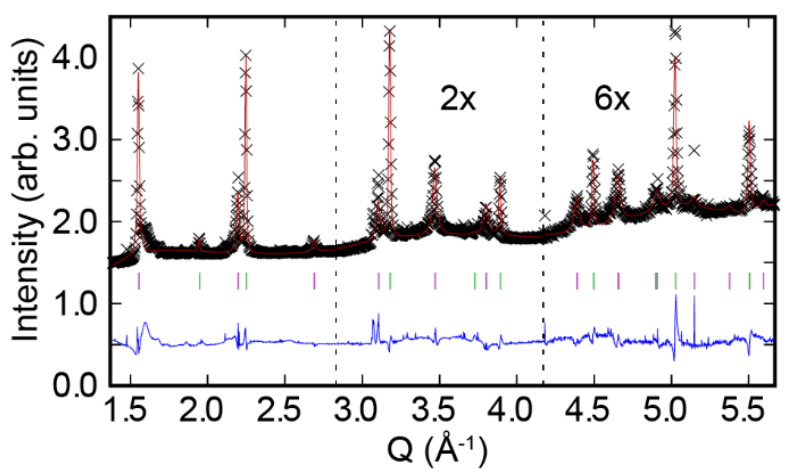

Figure S2. Rietveld fit to data from the high pressure $(\sim 0.69 \mathrm{GPa})$ experiment on cubic $\mathrm{LuZrF}_{7}$. The green tick marks indicate the positions of peaks from the $\mathrm{NaCl}$ pressure standard.

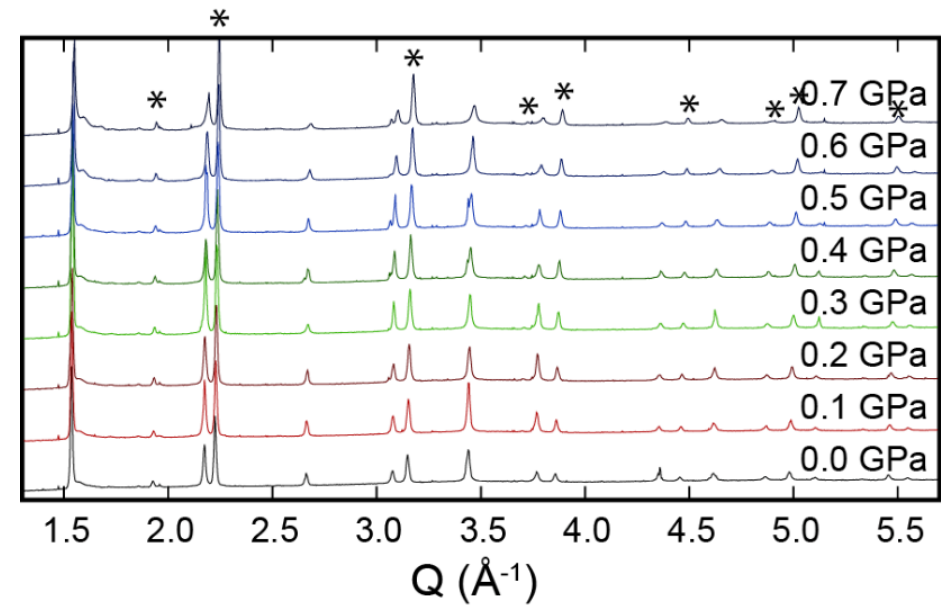

Figure S3. Selected patterns for $\mathrm{LuZrF}_{7}$ on compression. * peaks from the $\mathrm{NaCl}$ pressure calibrant. 


\section{Supplementary Tables}

Table S1. Crystallographic model for the Rietveld fit to the $302 \mathrm{~K}$ ambient pressure data from the Cryostream experiment.

\begin{tabular}{|l|l|}
\hline Chemical Formula & LuZrF $_{7}$ \\
\hline Space Group & $P m-3 m(\# 221)$ \\
\hline Lattice constant, a $(\AA)$ & $4.08263(4)$ \\
\hline X-ray wavelength $(\AA)$ & 0.45118 \\
\hline Pressure (GPa) & 0 \\
\hline Temperature (K) & 302 \\
\hline$R_{\text {wp }}$ as defined in GSAS & 0.0733 \\
\hline$R_{\mathrm{F}}{ }^{2}$ as defined in GSAS & 0.1119 \\
\hline
\end{tabular}

\begin{tabular}{|l|l|l|l|l|l|l|}
\hline $\begin{array}{l}\text { Atom and } \\
\text { Wyckoff } \\
\text { position }\end{array}$ & $\mathrm{x}$ & $\mathrm{y}$ & $\mathrm{z}$ & $\mathrm{U}_{\text {iso }}\left(\AA^{2}\right)$ & $\begin{array}{l}\text { Fractional } \\
\text { occupancy }\end{array}$ & $\begin{array}{l}\text { \# atoms } \\
\text { per unit } \\
\text { cell }\end{array}$ \\
\hline Lu 6e & 0 & 0 & $0.079(1)$ & $0.002(1)$ & 0.0833 & 0.50 \\
\hline Zr 6e & 0 & 0 & $0.079(1)$ & $0.002(1)$ & 0.0833 & 0.50 \\
\hline F1 12j & 0.5 & $0.059(1)$ & $0.059(1)$ & $0.019(2)$ & 0.2187 & 2.62 \\
\hline F2 24I & 0.5 & $0.293(5)$ & $0.120(5)$ & $0.019(2)$ & 0.0365 & 0.88 \\
\hline
\end{tabular}

Table S2. Crystallographic model for the Rietveld fit to the 0.35 GPa room temperature data from the high pressure experiment. The structural parameters were fixed at those obtained from the analysis of the ambient pressure data (see Table S1).

\begin{tabular}{|l|l|}
\hline Chemical Formula & LuZrF $_{7}$ \\
\hline Space Group & $P m-3 m(\# 221)$ \\
\hline Lattice constant, a $(\AA)$ & $4.0742(1)$ \\
\hline X-ray wavelength $(\AA)$ & 0.45118 \\
\hline Pressure $(\mathrm{GPa})$ & 0.35 \\
\hline Temperature $(\mathrm{K})$ & 295 \\
\hline $\mathrm{R}_{\mathrm{wp}}$ as defined in GSAS & 0.044 \\
\hline $\mathrm{R}_{\mathrm{F}}{ }^{2}$ as defined in GSAS & 0.114 \\
\hline
\end{tabular}

\begin{tabular}{|l|l|l|l|l|l|l|}
\hline $\begin{array}{l}\text { Atom and } \\
\text { Wyckoff } \\
\text { position }\end{array}$ & $\mathrm{x}$ & $\mathrm{y}$ & $\mathrm{z}$ & $\mathrm{U}_{\text {iso }}\left(\AA^{2}\right)$ & $\begin{array}{l}\text { Fractional } \\
\text { occupancy }\end{array}$ & $\begin{array}{l}\text { \# atoms } \\
\text { per unit } \\
\text { cell }\end{array}$ \\
\hline Lu 6e & 0 & 0 & 0.079 & 0.002 & 0.0833 & 0.50 \\
\hline Zr 6e & 0 & 0 & 0.079 & 0.002 & 0.0833 & 0.50 \\
\hline F1 12j & 0.5 & 0.059 & 0.059 & 0.019 & 0.2187 & 2.62 \\
\hline F2 24l & 0.5 & 0.293 & 0.120 & 0.019 & 0.0365 & 0.88 \\
\hline
\end{tabular}


Table S3. Lattice constant and unit cell volume as a function of temperature for cubic $\mathrm{LuZrF}_{7}$

\begin{tabular}{|c|c|c|c|}
\hline Temperature (K) & $\begin{array}{l}\text { Lattice constant } \\
\text { for } \operatorname{LuZrF}_{7}(\AA)\end{array}$ & $\begin{array}{l}\text { Unit cell Volume } \\
\text { for } \mathrm{LuZrF}_{7}\left(\AA^{3}\right)\end{array}$ & $\begin{array}{l}\text { Unit cell volume for silicon } \\
\text { internal standard }\left(\AA^{3}\right)\end{array}$ \\
\hline 101.1 & $4.08103(4)$ & $67.969(2)$ & $159.89(2)$ \\
\hline 104.3 & $4.08105(4)$ & $67.970(2)$ & $159.89(2)$ \\
\hline 107.3 & $4.08105(4)$ & $67.970(2)$ & $159.89(2)$ \\
\hline 110.3 & $4.08104(4)$ & $67.969(2)$ & $159.89(2)$ \\
\hline 113.3 & $4.08101(4)$ & $67.968(2)$ & $159.89(2)$ \\
\hline 116.2 & $4.08098(4)$ & $67.966(2)$ & $159.89(2)$ \\
\hline 119.2 & $4.08098(4)$ & $67.966(2)$ & $159.89(2)$ \\
\hline 122.2 & $4.08098(4)$ & $67.966(2)$ & $159.89(2)$ \\
\hline 125.2 & $4.08099(4)$ & $67.967(2)$ & $159.89(2)$ \\
\hline 128.2 & $4.08100(4)$ & $67.967(2)$ & $159.89(2)$ \\
\hline 131.1 & $4.08100(4)$ & $67.967(2)$ & $159.89(2)$ \\
\hline 134.2 & $4.08100(4)$ & $67.967(2)$ & $159.89(2)$ \\
\hline 137.2 & $4.08100(4)$ & $67.967(2)$ & $159.89(2)$ \\
\hline 140.1 & $4.08102(4)$ & $67.968(2)$ & $159.89(2)$ \\
\hline 143.1 & $4.08105(4)$ & $67.970(2)$ & $159.90(2)$ \\
\hline 146.1 & $4.08107(4)$ & $67.971(2)$ & $159.90(2)$ \\
\hline 149.1 & $4.08108(4)$ & $67.971(2)$ & $159.90(2)$ \\
\hline 152.1 & $4.08110(4)$ & $67.972(2)$ & $159.91(2)$ \\
\hline 155.1 & $4.08110(4)$ & $67.972(2)$ & $159.91(2)$ \\
\hline 158.1 & $4.08113(4)$ & $67.974(2)$ & $159.91(2)$ \\
\hline 161.1 & $4.08114(4)$ & $67.974(2)$ & $159.91(2)$ \\
\hline 164.0 & $4.08116(4)$ & $67.975(2)$ & $159.91(2)$ \\
\hline 167.1 & $4.08118(4)$ & $67.976(2)$ & $159.92(2)$ \\
\hline 170.0 & $4.08121(4)$ & $67.978(2)$ & $159.92(2)$ \\
\hline
\end{tabular}




\begin{tabular}{|c|c|c|c|}
\hline 173.1 & $4.08123(4)$ & $67.979(2)$ & $159.92(2)$ \\
\hline 176.1 & $4.08125(4)$ & $67.980(2)$ & $159.93(2)$ \\
\hline 179.0 & $4.08126(4)$ & $67.980(2)$ & $159.93(2)$ \\
\hline 182.1 & $4.08127(4)$ & $67.981(2)$ & $159.93(2)$ \\
\hline 185.0 & $4.08129(4)$ & $67.982(2)$ & $159.93(2)$ \\
\hline 188.0 & $4.08131(4)$ & $67.983(2)$ & $159.93(2)$ \\
\hline 191.0 & $4.08132(4)$ & $67.983(2)$ & $159.93(2)$ \\
\hline 194.1 & $4.08133(4)$ & $67.984(2)$ & $159.93(2)$ \\
\hline 197.0 & $4.08135(4)$ & $67.985(2)$ & $159.94(2)$ \\
\hline 200.0 & $4.08136(4)$ & $67.985(2)$ & $159.94(2)$ \\
\hline 203.1 & $4.08139(4)$ & $67.987(2)$ & $159.94(2)$ \\
\hline 206.0 & $4.08141(4)$ & $67.988(2)$ & $159.94(2)$ \\
\hline 209.1 & $4.08143(4)$ & $67.989(2)$ & $159.94(2)$ \\
\hline 212.0 & $4.08147(4)$ & $67.991(2)$ & $159.95(2)$ \\
\hline 215.0 & $4.08151(4)$ & $67.993(2)$ & $159.95(2)$ \\
\hline 218.0 & $4.08153(4)$ & $67.994(2)$ & $159.95(2)$ \\
\hline 221.0 & $4.08156(4)$ & $67.995(2)$ & $159.95(2)$ \\
\hline 224.0 & $4.08159(4)$ & $67.997(2)$ & $159.96(2)$ \\
\hline 227.0 & $4.08162(4)$ & $67.998(2)$ & $159.96(2)$ \\
\hline 230.0 & $4.08165(4)$ & $68.000(2)$ & $159.96(2)$ \\
\hline 233.0 & $4.08168(4)$ & $68.001(2)$ & $159.96(2)$ \\
\hline 236.0 & $4.08172(4)$ & $68.003(2)$ & $159.97(2)$ \\
\hline 239.1 & $4.08174(4)$ & $68.004(2)$ & $159.97(2)$ \\
\hline 242.0 & $4.08176(4)$ & $68.005(2)$ & $159.97(2)$ \\
\hline 245.0 & $4.08179(4)$ & $68.007(2)$ & $159.97(2)$ \\
\hline 248.0 & $4.08182(4)$ & $68.008(2)$ & $159.98(2)$ \\
\hline 251.0 & $4.08186(4)$ & $68.010(2)$ & $159.98(2)$ \\
\hline 254.0 & $4.08191(4)$ & $68.013(2)$ & $159.98(2)$ \\
\hline
\end{tabular}




\begin{tabular}{|c|c|c|c|}
\hline 257.0 & $4.08194(4)$ & $68.014(2)$ & $159.99(2)$ \\
\hline 260.0 & $4.08197(4)$ & $68.016(2)$ & $159.99(2)$ \\
\hline 263.0 & $4.08201(4)$ & $68.018(2)$ & $159.99(2)$ \\
\hline 266.0 & $4.08206(4)$ & $68.020(2)$ & $160.00(2)$ \\
\hline 269.0 & $4.08212(4)$ & $68.023(2)$ & $160.00(2)$ \\
\hline 272.0 & $4.08217(4)$ & $68.026(2)$ & $160.00(2)$ \\
\hline 275.0 & $4.08222(4)$ & $68.028(2)$ & $160.01(2)$ \\
\hline 278.0 & $4.08229(4)$ & $68.032(2)$ & $160.01(2)$ \\
\hline 281.0 & $4.08234(4)$ & $68.034(2)$ & $160.01(2)$ \\
\hline 284.0 & $4.08240(4)$ & $68.037(2)$ & $160.02(2)$ \\
\hline 287.0 & $4.08246(4)$ & $68.040(2)$ & $160.02(2)$ \\
\hline 290.0 & $4.08252(4)$ & $68.043(2)$ & $160.02(2)$ \\
\hline 293.0 & $4.08258(4)$ & $68.046(2)$ & $160.03(2)$ \\
\hline 296.0 & $4.08264(4)$ & $68.049(2)$ & $160.03(2)$ \\
\hline 299.0 & $4.08269(4)$ & $68.052(2)$ & $160.04(2)$ \\
\hline 302.0 & $4.08277(4)$ & $68.056(2)$ & $160.04(2)$ \\
\hline 305.0 & $4.08283(4)$ & $68.059(2)$ & $160.04(2)$ \\
\hline 308.0 & $4.08290(4)$ & $68.062(2)$ & $160.05(2)$ \\
\hline 311.0 & $4.08296(4)$ & $68.065(2)$ & $160.05(2)$ \\
\hline 314.0 & $4.08302(4)$ & $68.068(2)$ & $160.05(2)$ \\
\hline 317.0 & $4.08309(4)$ & $68.072(2)$ & $160.06(2)$ \\
\hline 320.0 & $4.08316(4)$ & $68.075(2)$ & $160.06(2)$ \\
\hline 323.0 & $4.08322(4)$ & $68.078(2)$ & $160.07(2)$ \\
\hline 326.0 & $4.08328(4)$ & $68.081(2)$ & $160.07(2)$ \\
\hline 329.0 & $4.08334(4)$ & $68.084(2)$ & $160.08(2)$ \\
\hline 332.0 & $4.08340(4)$ & $68.087(2)$ & $160.08(2)$ \\
\hline 335.0 & $4.08346(4)$ & $68.090(2)$ & $160.08(2)$ \\
\hline 338.0 & $4.08352(4)$ & $68.093(2)$ & $160.09(2)$ \\
\hline
\end{tabular}




\begin{tabular}{|c|c|c|c|}
\hline 341.0 & $4.08359(4)$ & $68.097(2)$ & $160.09(2)$ \\
\hline 344.0 & $4.08365(4)$ & $68.100(2)$ & $160.10(2)$ \\
\hline 347.0 & $4.08373(4)$ & $68.104(2)$ & $160.10(2)$ \\
\hline 350.0 & $4.08379(4)$ & $68.107(2)$ & $160.11(2)$ \\
\hline 353.0 & $4.08386(4)$ & $68.110(2)$ & $160.11(2)$ \\
\hline 356.0 & $4.08394(4)$ & $68.114(2)$ & $160.11(2)$ \\
\hline 359.0 & $4.08400(4)$ & $68.117(2)$ & $160.12(2)$ \\
\hline 362.0 & $4.08408(4)$ & $68.121(2)$ & $160.12(2)$ \\
\hline 365.0 & $4.08416(4)$ & $68.125(2)$ & $160.13(2)$ \\
\hline 368.0 & $4.08423(4)$ & $68.129(2)$ & $160.13(2)$ \\
\hline 371.0 & $4.08431(4)$ & $68.133(2)$ & $160.14(2)$ \\
\hline 374.0 & $4.08438(4)$ & $68.136(2)$ & $160.14(2)$ \\
\hline 377.0 & $4.08448(4)$ & $68.141(2)$ & $160.15(2)$ \\
\hline 380.0 & $4.08458(4)$ & $68.146(2)$ & $160.15(2)$ \\
\hline 383.0 & $4.08468(4)$ & $68.151(2)$ & $160.16(2)$ \\
\hline 386.0 & $4.08479(4)$ & $68.157(2)$ & $160.16(2)$ \\
\hline 389.0 & $4.08490(4)$ & $68.162(2)$ & $160.17(2)$ \\
\hline 392.0 & $4.08503(4)$ & $68.169(2)$ & $160.17(2)$ \\
\hline 395.0 & $4.08513(4)$ & $68.174(2)$ & $160.17(2)$ \\
\hline 398.0 & $4.08525(4)$ & $68.180(2)$ & $160.18(2)$ \\
\hline 401.0 & $4.08537(4)$ & $68.186(2)$ & $160.18(2)$ \\
\hline 404.1 & $4.08548(4)$ & $68.191(2)$ & $160.19(2)$ \\
\hline 407.0 & $4.08558(4)$ & $68.196(2)$ & $160.19(2)$ \\
\hline 410.0 & $4.08569(4)$ & $68.202(2)$ & $160.20(2)$ \\
\hline 413.0 & $4.08580(4)$ & $68.207(2)$ & $160.20(2)$ \\
\hline 416.0 & 4.08591(4) & $68.213(2)$ & $160.21(2)$ \\
\hline 419.0 & $4.08602(4)$ & $68.218(2)$ & $160.21(2)$ \\
\hline 422.0 & $4.08612(4)$ & $68.223(2)$ & $160.22(2)$ \\
\hline
\end{tabular}




\begin{tabular}{|c|c|c|c|}
\hline 425.0 & $4.08623(4)$ & $68.229(2)$ & $160.22(2)$ \\
\hline 428.0 & $4.08634(4)$ & $68.234(2)$ & $160.23(2)$ \\
\hline 431.0 & $4.08640(4)$ & $68.237(2)$ & $160.23(2)$ \\
\hline 434.0 & $4.08654(4)$ & $68.244(2)$ & $160.24(2)$ \\
\hline 437.0 & $4.08660(4)$ & $68.247(2)$ & $160.24(2)$ \\
\hline 440.0 & $4.08671(4)$ & $68.253(2)$ & $160.25(2)$ \\
\hline 443.0 & $4.08681(4)$ & $68.258(2)$ & $160.25(2)$ \\
\hline 446.0 & $4.08692(4)$ & $68.263(2)$ & $160.26(2)$ \\
\hline 449.0 & $4.08702(4)$ & $68.268(2)$ & $160.26(2)$ \\
\hline 452.0 & $4.08710(4)$ & $68.272(2)$ & $160.27(2)$ \\
\hline 455.0 & $4.08719(4)$ & $68.277(2)$ & $160.27(2)$ \\
\hline 458.0 & $4.08728(4)$ & $68.282(2)$ & $160.28(2)$ \\
\hline 461.0 & $4.08736(4)$ & $68.286(2)$ & $160.28(2)$ \\
\hline 464.0 & $4.08744(4)$ & $68.290(2)$ & $160.29(2)$ \\
\hline 467.0 & $4.08751(4)$ & $68.293(2)$ & $160.29(2)$ \\
\hline 470.0 & $4.08759(4)$ & $68.297(2)$ & $160.30(2)$ \\
\hline 473.1 & $4.08766(4)$ & $68.301(2)$ & $160.30(2)$ \\
\hline 476.0 & $4.08775(4)$ & $68.305(2)$ & $160.31(2)$ \\
\hline 479.0 & $4.08783(4)$ & $68.309(2)$ & $160.31(2)$ \\
\hline 482.0 & $4.08792(4)$ & $68.314(2)$ & $160.32(2)$ \\
\hline 485.0 & $4.08801(4)$ & $68.318(2)$ & $160.32(2)$ \\
\hline 488.0 & $4.08810(4)$ & $68.323(2)$ & $160.32(2)$ \\
\hline 491.0 & $4.08819(4)$ & $68.327(2)$ & $160.33(2)$ \\
\hline 494.1 & $4.08828(4)$ & $68.332(2)$ & $160.34(2)$ \\
\hline 497.0 & $4.08839(4)$ & $68.337(2)$ & $160.34(2)$ \\
\hline 500.0 & $4.08866(4)$ & $68.351(2)$ & $160.34(2)$ \\
\hline 500.0 & $4.08867(4)$ & $68.351(2)$ & $160.34(2)$ \\
\hline 497.0 & $4.08863(4)$ & $68.349(2)$ & $160.34(2)$ \\
\hline
\end{tabular}




\begin{tabular}{|c|c|c|c|}
\hline 494.0 & $4.08860(4)$ & $68.348(2)$ & $160.33(2)$ \\
\hline 491.0 & $4.08858(4)$ & $68.347(2)$ & $160.33(2)$ \\
\hline 487.9 & $4.08854(4)$ & $68.345(2)$ & $160.32(2)$ \\
\hline 485.0 & $4.08851(4)$ & $68.343(2)$ & $160.32(2)$ \\
\hline 482.0 & $4.08846(4)$ & $68.341(2)$ & $160.31(2)$ \\
\hline 478.9 & $4.088410(4)$ & $68.338(2)$ & $160.31(2)$ \\
\hline 476.0 & $4.08837(4)$ & $68.336(2)$ & $160.30(2)$ \\
\hline 473.0 & $4.08831(4)$ & $68.333(2)$ & $160.30(2)$ \\
\hline 469.9 & $4.08827(4)$ & $68.331(2)$ & $160.29(2)$ \\
\hline 467.0 & $4.08820(4)$ & $68.328(2)$ & $160.29(2)$ \\
\hline 464.0 & $4.08814(4)$ & $68.325(2)$ & $160.28(2)$ \\
\hline 460.9 & $4.08804(4)$ & $68.320(2)$ & $160.27(2)$ \\
\hline 457.9 & $4.08795(4)$ & $68.315(2)$ & $160.27(2)$ \\
\hline 455.0 & $4.08789(4)$ & $68.312(2)$ & $160.26(2)$ \\
\hline 452.0 & $4.08781(4)$ & $68.308(2)$ & $160.26(2)$ \\
\hline 448.9 & $4.08774(4)$ & $68.305(2)$ & $160.25(2)$ \\
\hline 446.0 & $4.08766(4)$ & $68.301(2)$ & $160.24(2)$ \\
\hline 443.0 & $4.08759(4)$ & $68.297(2)$ & $160.24(2)$ \\
\hline 440.0 & $4.08751(4)$ & $68.293(2)$ & $160.23(2)$ \\
\hline 437.0 & $4.08743(4)$ & $68.289(2)$ & $160.23(2)$ \\
\hline 434.0 & $4.08735(4)$ & $68.285(2)$ & $160.22(2)$ \\
\hline 431.0 & $4.08727(4)$ & $68.281(2)$ & $160.22(2)$ \\
\hline 427.9 & $4.08718(4)$ & $68.277(2)$ & $160.21(2)$ \\
\hline 425.0 & $4.08710(4)$ & $68.272(2)$ & $160.21(2)$ \\
\hline 421.9 & $4.08701(4)$ & $68.268(2)$ & $160.20(2)$ \\
\hline 419.0 & $4.08691(4)$ & $68.263(2)$ & $160.20(2)$ \\
\hline 416.0 & $4.08682(4)$ & $68.258(2)$ & $160.19(2)$ \\
\hline 412.9 & $4.08672(4)$ & $68.253(2)$ & $160.19(2)$ \\
\hline
\end{tabular}




\begin{tabular}{|c|c|c|c|}
\hline 410.0 & $4.08664(4)$ & $68.249(2)$ & $160.19(2)$ \\
\hline 407.0 & $4.08653(4)$ & $68.244(2)$ & $160.18(2)$ \\
\hline 403.0 & $4.08643(4)$ & $68.239(2)$ & $160.18(2)$ \\
\hline 401.0 & $4.08633(4)$ & $68.234(2)$ & $160.17(2)$ \\
\hline 398.0 & $4.08621(4)$ & $68.228(2)$ & $160.17(2)$ \\
\hline 394.9 & $4.08610(4)$ & $68.222(2)$ & $160.16(2)$ \\
\hline 392.0 & $4.08599(4)$ & $68.217(2)$ & $160.16(2)$ \\
\hline 389.0 & $4.08588(4)$ & $68.211(2)$ & $160.15(2)$ \\
\hline 386.0 & $4.08577(4)$ & $68.206(2)$ & $160.15(2)$ \\
\hline 383.0 & $4.08565(4)$ & $68.200(2)$ & $160.14(2)$ \\
\hline 380.0 & $4.08553(4)$ & $68.194(2)$ & $160.14(2)$ \\
\hline 377.0 & $4.08541(4)$ & $68.188(2)$ & $160.13(2)$ \\
\hline 374.0 & $4.08529(4)$ & $68.182(2)$ & $160.13(2)$ \\
\hline 370.9 & $4.08516(4)$ & $68.175(2)$ & $160.12(2)$ \\
\hline 368.0 & $4.08504(4)$ & $68.169(2)$ & $160.12(2)$ \\
\hline 365.0 & $4.08492(4)$ & $68.163(2)$ & $160.12(2)$ \\
\hline 362.0 & $4.08481(4)$ & $68.158(2)$ & $160.11(2)$ \\
\hline 359.0 & $4.08468(4)$ & $68.151(2)$ & $160.11(2)$ \\
\hline 356.0 & $4.08457(4)$ & $68.146(2)$ & $160.10(2)$ \\
\hline 353.0 & $4.08444(4)$ & $68.139(2)$ & $160.10(2)$ \\
\hline 350.0 & $4.08433(4)$ & $68.134(2)$ & $160.09(2)$ \\
\hline 347.0 & $4.08422(4)$ & $68.128(2)$ & $160.09(2)$ \\
\hline 344.0 & $4.08411(4)$ & $68.123(2)$ & $160.08(2)$ \\
\hline 341.0 & $4.08400(4)$ & $68.117(2)$ & $160.08(2)$ \\
\hline 338.0 & $4.08390(4)$ & $68.112(2)$ & $160.07(2)$ \\
\hline 334.9 & $4.08380(4)$ & $68.107(2)$ & $160.07(2)$ \\
\hline 331.9 & $4.08370(4)$ & $68.102(2)$ & $160.07(2)$ \\
\hline 329.0 & $4.08359(4)$ & $68.097(2)$ & $160.06(2)$ \\
\hline
\end{tabular}




\begin{tabular}{|c|c|c|c|}
\hline 326.0 & $4.08352(4)$ & $68.093(2)$ & $160.06(2)$ \\
\hline 323.0 & $4.08343(4)$ & $68.089(2)$ & $160.05(2)$ \\
\hline 320.0 & $4.08335(4)$ & $68.085(2)$ & $160.05(2)$ \\
\hline 316.9 & $4.08327(4)$ & $68.081(2)$ & $160.05(2)$ \\
\hline 313.9 & $4.08321(4)$ & $68.078(2)$ & $160.04(2)$ \\
\hline 310.9 & $4.08315(4)$ & $68.075(2)$ & $160.04(2)$ \\
\hline 308.0 & $4.08304(4)$ & $68.069(2)$ & $160.04(2)$ \\
\hline 304.9 & $4.08294(4)$ & $68.064(2)$ & $160.03(2)$ \\
\hline 302.0 & $4.08286(4)$ & $68.060(2)$ & $160.02(2)$ \\
\hline 298.9 & $4.08278(4)$ & $68.056(2)$ & $160.02(2)$ \\
\hline 295.9 & $4.08270(4)$ & $68.052(2)$ & $160.02(2)$ \\
\hline 293.0 & $4.08262(4)$ & $68.048(2)$ & $160.01(2)$ \\
\hline 289.9 & $4.08256(4)$ & $68.045(2)$ & $160.01(2)$ \\
\hline 287.0 & $4.08249(4)$ & $68.042(2)$ & $160.01(2)$ \\
\hline 283.9 & $4.08243(4)$ & $68.039(2)$ & $160.00(2)$ \\
\hline 280.9 & $4.08238(4)$ & $68.036(2)$ & $160.00(2)$ \\
\hline 278.0 & $4.08233(4)$ & $68.034(2)$ & $160.00(2)$ \\
\hline 274.9 & $4.08229(4)$ & $68.032(2)$ & $159.99(2)$ \\
\hline 272.0 & $4.08227(4)$ & $68.031(2)$ & $159.99(2)$ \\
\hline 269.0 & $4.08223(4)$ & $68.029(2)$ & $159.99(2)$ \\
\hline 265.9 & $4.08216(4)$ & $68.025(2)$ & $159.99(2)$ \\
\hline 262.9 & $4.08210(4)$ & $68.022(2)$ & $159.98(2)$ \\
\hline 259.9 & $4.08205(4)$ & $68.020(2)$ & $159.97(2)$ \\
\hline 256.9 & $4.08201(4)$ & $68.018(2)$ & $159.97(2)$ \\
\hline 253.9 & $4.08197(4)$ & $68.016(2)$ & $159.97(2)$ \\
\hline 250.9 & $4.08193(4)$ & $68.014(2)$ & $159.97(2)$ \\
\hline 247.9 & $4.08190(4)$ & $68.012(2)$ & $159.96(2)$ \\
\hline 244.9 & $4.08186(4)$ & $68.010(2)$ & $159.96(2)$ \\
\hline
\end{tabular}




\begin{tabular}{|c|c|c|c|}
\hline 241.9 & $4.08184(4)$ & $68.009(2)$ & $159.96(2)$ \\
\hline 238.9 & $4.08184(4)$ & $68.009(2)$ & $159.96(2)$ \\
\hline 235.9 & $4.08181(4)$ & $68.008(2)$ & $159.96(2)$ \\
\hline 232.9 & $4.08178(4)$ & $68.006(2)$ & $159.95(2)$ \\
\hline 229.9 & $4.08172(4)$ & $68.003(2)$ & $159.95(2)$ \\
\hline 226.9 & $4.08168(4)$ & $68.001(2)$ & $159.94(2)$ \\
\hline 223.9 & $4.08166(4)$ & $68.000(2)$ & $159.94(2)$ \\
\hline 221.0 & $4.08161(4)$ & $67.998(2)$ & $159.94(2)$ \\
\hline 217.9 & $4.08159(4)$ & $67.997(2)$ & $159.94(2)$ \\
\hline 211.9 & $4.08158(4)$ & $67.996(2)$ & $159.93(2)$ \\
\hline 208.9 & $4.08158(4)$ & $67.996(2)$ & $159.93(2)$ \\
\hline 205.9 & $4.08158(4)$ & $67.996(2)$ & $159.94(2)$ \\
\hline 202.9 & $4.08158(4)$ & $67.996(2)$ & $159.93(2)$ \\
\hline 199.9 & $4.08153(4)$ & $67.994(2)$ & $159.93(2)$ \\
\hline 196.9 & $4.08149(4)$ & $67.992(2)$ & $159.92(2)$ \\
\hline 193.9 & $4.08148(4)$ & $67.991(2)$ & $159.92(2)$ \\
\hline 190.9 & $4.08146(4)$ & $67.990(2)$ & $159.92(2)$ \\
\hline 187.9 & $4.08146(4)$ & $67.990(2)$ & $159.92(2)$ \\
\hline 184.8 & $4.08145(4)$ & $67.990(2)$ & $159.92(2)$ \\
\hline 181.8 & $4.08147(4)$ & $67.991(2)$ & $159.92(2)$ \\
\hline 178.8 & $4.08147(4)$ & $67.991(2)$ & $159.92(2)$ \\
\hline 175.8 & $4.08147(4)$ & $67.991(2)$ & $159.92(2)$ \\
\hline 172.8 & $4.08144(4)$ & $67.989(2)$ & $159.91(2)$ \\
\hline 169.7 & $4.08140(4)$ & $67.987(2)$ & $159.91(2)$ \\
\hline 166.7 & $4.08139(4)$ & $67.987(2)$ & $159.91(2)$ \\
\hline 163.7 & $4.08139(4)$ & $67.987(2)$ & $159.91(2)$ \\
\hline 160.6 & $4.08139(4)$ & $67.987(2)$ & $159.90(2)$ \\
\hline 157.6 & $4.08141(4)$ & $67.988(2)$ & 159.91(2) \\
\hline
\end{tabular}




\begin{tabular}{|c|c|c|c|}
\hline 154.5 & $4.08141(4)$ & $67.988(2)$ & $159.91(2)$ \\
\hline 151.5 & $4.08142(4)$ & $67.988(2)$ & $159.91(2)$ \\
\hline 148.4 & $4.08142(4)$ & $67.988(2)$ & $159.90(2)$ \\
\hline 145.2 & $4.08136(4)$ & $67.985(2)$ & $159.90(2)$ \\
\hline 142.2 & $4.08136(4)$ & $67.985(2)$ & $159.90(2)$ \\
\hline 139.1 & $4.08136(4)$ & $67.985(2)$ & $159.90(2)$ \\
\hline 136.2 & $4.08136(4)$ & $67.985(2)$ & $159.90(2)$ \\
\hline 133.2 & $4.08136(4)$ & $67.985(2)$ & $159.90(2)$ \\
\hline 130.2 & $4.08139(4)$ & $67.987(2)$ & $159.90(2)$ \\
\hline 127.3 & $4.08141(4)$ & $67.988(2)$ & $159.90(2)$ \\
\hline 124.4 & $4.08141(4)$ & $67.988(2)$ & $159.90(2)$ \\
\hline 121.6 & $4.08141(4)$ & $67.988(2)$ & $159.90(2)$ \\
\hline 118.8 & $4.08141(4)$ & $67.988(2)$ & $159.90(2)$ \\
\hline 116.0 & $4.08138(4)$ & $67.986(2)$ & $159.90(2)$ \\
\hline 113.2 & $4.08138(4)$ & $67.986(2)$ & $159.89(2)$ \\
\hline 110.3 & $4.08140(4)$ & $67.987(2)$ & $159.89(2)$ \\
\hline 108.0 & $4.08140(4)$ & $67.987(2)$ & $159.89(2)$ \\
\hline 105.0 & $4.08141(4)$ & $67.988(2)$ & $159.89(2)$ \\
\hline 102.5 & $4.08144(4)$ & $67.989(2)$ & $159.90(2)$ \\
\hline 101.1 & $4.08143(4)$ & $67.989(2)$ & $159.90(2)$ \\
\hline
\end{tabular}


Table S4. Lattice constant and unit cell volume as a function of pressure for cubic $\mathrm{LuZrF}_{7}$

\begin{tabular}{|c|c|c|c|}
\hline Pressure (GPa) & $\begin{array}{l}\text { Lattice constant for } \\
\text { cubic } \operatorname{LuZrF}_{7}(\AA \AA)\end{array}$ & $\begin{array}{l}\text { Unit cell volume for } \\
\text { cubic } \operatorname{LuZrF}_{7}\left(\AA^{3}\right)\end{array}$ & $\begin{array}{l}\text { Unit cell volume for the } \\
\mathrm{NaCl} \text { pressure calibrant }\left(\AA^{3}\right)\end{array}$ \\
\hline 0.00 & $4.0845(1)$ & $68.144(6)$ & $179.85(2)$ \\
\hline 0.00 & $4.0846(1)$ & $68.149(6)$ & $179.85(2)$ \\
\hline 0.00 & $4.0846(1)$ & $68.145(7)$ & $179.85(2)$ \\
\hline 0.00 & $4.0843(1)$ & $68.132(7)$ & $179.85(2)$ \\
\hline 0.00 & $4.0834(1)$ & $68.085(7)$ & $179.85(2)$ \\
\hline 0.00 & $4.0843(1)$ & $68.135(7)$ & $179.85(2)$ \\
\hline 0.00 & $4.0849(1)$ & $68.160(7)$ & $179.85(2)$ \\
\hline 0.00 & 4.0849(1) & $68.160(6)$ & $179.85(1)$ \\
\hline 0.00 & $4.0852(1)$ & $68.179(7)$ & $179.85(2)$ \\
\hline 0.00 & $4.0858(1)$ & $68.208(7)$ & $179.85(2)$ \\
\hline 0.00 & $4.0867(2)$ & $68.252(9)$ & $179.84(2)$ \\
\hline 0.00 & $4.0883(2)$ & 68.33(1) & $179.85(2)$ \\
\hline 0.00 & $4.0867(2)$ & $68.254(9)$ & $179.85(2)$ \\
\hline 0.00 & $4.0872(1)$ & $68.277(7)$ & $179.85(2)$ \\
\hline 0.00 & $4.0866(1)$ & $68.247(6)$ & $179.85(1)$ \\
\hline 0.00 & $4.0871(1)$ & $68.275(6)$ & $179.85(1)$ \\
\hline 0.00 & $4.0860(1)$ & $68.218(6)$ & $179.85(1)$ \\
\hline 0.00 & $4.0876(1)$ & $68.295(7)$ & $179.84(2)$ \\
\hline 0.00 & $4.0858(1)$ & $68.207(5)$ & $179.83(1)$ \\
\hline 0.00 & $4.0875(2)$ & $68.294(8)$ & $179.83(2)$ \\
\hline 0.00 & $4.0868(1)$ & $68.259(7)$ & $179.83(2)$ \\
\hline 0.00 & $4.0862(1)$ & $68.227(6)$ & $179.83(2)$ \\
\hline 0.01 & $4.0874(1)$ & $68.290(7)$ & $179.82(2)$ \\
\hline 0.01 & $4.0895(2)$ & $68.40(1)$ & $179.81(2)$ \\
\hline 0.01 & $4.0860(1)$ & $68.216(7)$ & $179.81(2)$ \\
\hline
\end{tabular}




\begin{tabular}{|c|c|c|c|}
\hline 0.01 & $4.0857(1)$ & $68.205(5)$ & $179.81(1)$ \\
\hline 0.01 & $4.0874(1)$ & $68.290(6)$ & $179.80(1)$ \\
\hline 0.01 & $4.0862(1)$ & $68.230(5)$ & 179.78(1) \\
\hline 0.01 & $4.0865(1)$ & $68.240(6)$ & $179.77(2)$ \\
\hline 0.01 & $4.0864(1)$ & $68.235(5)$ & $179.79(2)$ \\
\hline 0.01 & $4.0861(1)$ & $68.222(5)$ & $179.80(2)$ \\
\hline 0.01 & $4.0861(1)$ & $68.221(5)$ & $179.82(2)$ \\
\hline 0.01 & $4.08610(9)$ & $68.223(5)$ & $179.81(2)$ \\
\hline 0.01 & $4.0864(1)$ & $68.235(6)$ & $179.80(2)$ \\
\hline 0.01 & $4.0860(1)$ & $68.216(5)$ & $179.81(1)$ \\
\hline 0.01 & $4.0854(1)$ & $68.186(5)$ & $179.82(2)$ \\
\hline 0.00 & $4.08559(9)$ & $68.197(5)$ & $179.83(1)$ \\
\hline 0.00 & $4.0869(1)$ & $68.262(5)$ & $179.85(2)$ \\
\hline 0.00 & $4.0862(1)$ & $68.228(5)$ & $179.85(2)$ \\
\hline 0.00 & $4.0856(1)$ & $68.196(5)$ & $179.86(2)$ \\
\hline 0.00 & $4.0868(1)$ & $68.259(5)$ & $179.83(2)$ \\
\hline 0.01 & $4.0868(1)$ & $68.257(7)$ & $179.81(2)$ \\
\hline 0.01 & $4.0857(1)$ & $68.202(6)$ & $179.78(2)$ \\
\hline 0.01 & $4.08612(9)$ & $68.224(5)$ & $179.76(2)$ \\
\hline 0.02 & $4.0857(1)$ & $68.204(5)$ & $179.74(2)$ \\
\hline 0.02 & $4.0862(1)$ & $68.227(6)$ & $179.72(2)$ \\
\hline 0.02 & $4.08551(9)$ & $68.193(4)$ & 179.68(1) \\
\hline 0.03 & $4.0848(1)$ & $68.157(5)$ & $179.64(2)$ \\
\hline 0.03 & $4.0848(1)$ & $68.159(5)$ & $179.60(2)$ \\
\hline 0.04 & $4.0848(1)$ & $68.156(7)$ & $179.55(2)$ \\
\hline 0.05 & $4.0844(1)$ & $68.135(5)$ & $179.52(2)$ \\
\hline 0.05 & $4.0852(1)$ & $68.179(5)$ & $179.48(2)$ \\
\hline 0.06 & $4.0841(1)$ & $68.123(5)$ & $179.44(2)$ \\
\hline
\end{tabular}




\begin{tabular}{|c|c|c|c|}
\hline 0.06 & $4.08446(9)$ & $68.140(4)$ & $179.40(1)$ \\
\hline 0.07 & $4.0847(1)$ & $68.150(6)$ & $179.35(1)$ \\
\hline 0.08 & $4.0854(1)$ & $68.189(7)$ & $179.30(2)$ \\
\hline 0.08 & $4.0843(1)$ & $68.130(5)$ & $179.26(2)$ \\
\hline 0.09 & $4.08345(9)$ & $68.090(5)$ & $179.21(2)$ \\
\hline 0.09 & $4.0837(1)$ & $68.103(5)$ & $179.16(2)$ \\
\hline 0.10 & $4.0833(1)$ & $68.082(5)$ & $179.12(2)$ \\
\hline 0.10 & $4.0830(1)$ & $68.065(5)$ & $179.09(2)$ \\
\hline 0.11 & $4.0828(1)$ & $68.055(6)$ & $179.05(2)$ \\
\hline 0.12 & $4.0832(1)$ & $68.078(5)$ & $178.99(2)$ \\
\hline 0.12 & $4.0830(1)$ & $68.069(5)$ & $178.96(2)$ \\
\hline 0.13 & $4.0824(1)$ & $68.035(5)$ & $178.92(2)$ \\
\hline 0.13 & $4.0822(1)$ & $68.025(6)$ & $178.90(2)$ \\
\hline 0.13 & $4.0817(1)$ & $68.000(6)$ & $178.86(2)$ \\
\hline 0.14 & $4.0818(1)$ & $68.007(5)$ & $178.80(2)$ \\
\hline 0.15 & $4.0811(1)$ & $67.973(5)$ & $178.75(2)$ \\
\hline 0.16 & $4.0813(1)$ & $67.980(5)$ & $178.70(1)$ \\
\hline 0.17 & $4.0808(1)$ & $67.956(5)$ & $178.59(2)$ \\
\hline 0.18 & $4.0804(1)$ & $67.939(6)$ & $178.51(2)$ \\
\hline 0.19 & $4.0803(1)$ & $67.934(6)$ & $178.45(2)$ \\
\hline 0.20 & $4.0822(2)$ & $68.027(8)$ & $178.38(2)$ \\
\hline 0.21 & $4.0815(1)$ & $67.992(7)$ & $178.30(2)$ \\
\hline 0.22 & $4.0791(1)$ & $67.874(6)$ & $178.22(2)$ \\
\hline 0.24 & $4.0780(1)$ & $67.819(5)$ & 178.13(1) \\
\hline 0.25 & $4.0773(1)$ & $67.780(5)$ & $178.06(2)$ \\
\hline 0.26 & $4.0776(1)$ & $67.796(5)$ & $177.98(2)$ \\
\hline 0.27 & $4.0772(1)$ & $67.776(5)$ & $177.88(2)$ \\
\hline 0.28 & $4.0766(1)$ & $67.746(5)$ & $177.80(2)$ \\
\hline
\end{tabular}




\begin{tabular}{|c|c|c|c|}
\hline 0.29 & $4.0758(1)$ & $67.709(5)$ & $177.74(2)$ \\
\hline 0.30 & $4.0754(1)$ & $67.685(5)$ & $177.65(2)$ \\
\hline 0.32 & $4.0749(1)$ & $67.661(6)$ & $177.54(2)$ \\
\hline 0.34 & $4.0744(1)$ & $67.636(6)$ & $177.43(2)$ \\
\hline 0.35 & $4.0742(1)$ & $67.630(6)$ & $177.35(2)$ \\
\hline 0.36 & $4.0737(1)$ & $67.600(5)$ & $177.23(2)$ \\
\hline 0.38 & $4.0735(1)$ & $67.593(6)$ & $177.10(2)$ \\
\hline 0.40 & $4.0729(1)$ & $67.565(6)$ & $176.98(2)$ \\
\hline 0.42 & $4.0722(1)$ & $67.526(7)$ & $176.89(2)$ \\
\hline 0.44 & $4.0715(1)$ & $67.495(7)$ & $176.73(2)$ \\
\hline 0.46 & $4.0697(1)$ & $67.405(6)$ & $176.57(2)$ \\
\hline 0.48 & $4.0687(1)$ & $67.357(6)$ & $176.42(2)$ \\
\hline 0.50 & $4.0678(1)$ & $67.310(7)$ & $176.30(2)$ \\
\hline 0.52 & $4.0669(1)$ & $67.265(7)$ & $176.21(2)$ \\
\hline 0.54 & $4.0648(1)$ & $67.163(7)$ & $176.08(2)$ \\
\hline 0.56 & $4.0630(1)$ & $67.073(6)$ & $175.92(2)$ \\
\hline 0.58 & $4.0618(1)$ & $67.010(7)$ & $175.81(2)$ \\
\hline 0.59 & $4.0608(1)$ & $66.964(7)$ & $175.72(2)$ \\
\hline 0.60 & $4.0596(1)$ & $66.901(7)$ & $175.63(2)$ \\
\hline 0.64 & $4.0571(2)$ & $66.782(9)$ & $175.41(2)$ \\
\hline 0.67 & $4.0544(2)$ & $66.65(1)$ & $175.22(2)$ \\
\hline 0.69 & $4.0507(2)$ & $66.47(1)$ & $175.05(2)$ \\
\hline
\end{tabular}

